Puesta en funcionamiento del Área Anticorrupción y Manual de Procedimiento en la Universidad Nacional de La Plata

Melisa J. Fresco

Revista ES (en y sobre Educación Superior)

Vol.1, N¹-2 / Fecha de publicación: 27/12/2021

e-ISSN: $2718-6539$

https://revistas.unlp.edu.ar/ES/index

IIES - Facultad de Odontología

DOI: https://doi.org/10.24215/27186539e045

\title{
Puesta en funcionamiento del Área Anticorrupción y Manual de Procedimiento en la Universidad Nacional de La Plata
}

Commissioning of the Anti-Corruption Area and Procedure Manual at the National University of La Plata

Comissionamento da Área Anticorrupção e Manual de Procedimentos da Universidade Nacional de La Plata

\author{
Abogada Melisa J. Fresco \\ melisa.fresco@presi.unlp.edu.ar
}

\section{Resumen}

Con el objeto de preservar los altos estándares que hacen de nuestra universidad una de las más prestigiosas del país, es que resulta de gran relevancia pensar en delimitar márgenes de transparencia y lucha contra la 
corrupción, en donde encontraremos normas ya establecidas y algunos campos por trabajar.

La meta principal de este trabajo integrador es crear en el ámbito universitario parámetros y procedimientos de temas que hoy se encuentran en auge y nuestra institución le da tratamiento pero de manera aislada y sin un procedimiento uniforme para toda la universidad.

Sin embargo, previo a establecer procedimientos y a crear un manual completo del tema a tratar, será necesario realizar un estudio de los conceptos generales en la materia que emanan de convenciones internacionales y leyes nacionales; debiendo también analizar la normativa existente en nuestra universidad a fin de que podamos sobre su base trabajar los puntos que sean de relevancia.

\section{Abstract}

In order to preserve the high standards that make our university one of the most prestigious in the country, it is highly relevant to think about defining margins of transparency and the fight against corruption, where we will find already established standards and some fields to work on. .

The main goal of this integrative work is to create parameters and procedures of topics that are currently on the rise in the university environment and our institution treats them but in an isolated manner and without a uniform procedure for the entire university.

However, prior to establishing procedures and creating a complete manual on the subject to be dealt with, it will be necessary to carry out a study of the general concepts in the matter that emanate from international conventions and national laws; also having to analyze the existing regulations in our university so that we can work on the relevant points on their basis. 


\section{Resumo}

Para preservar os elevados padrões que fazem da nossa universidade uma das mais prestigiadas do país, é muito relevante pensar em definir margens de transparência e de combate à corrupção, onde encontraremos padrões já estabelecidos e alguns campos de atuação.

O objetivo principal deste trabalho integrativo é criar parâmetros e procedimentos de temas que atualmente estão em alta no ambiente universitário e nossa instituição os trata, mas de forma isolada e sem um procedimento uniforme para toda a universidade.

Porém, antes de estabelecer procedimentos e elaborar um manual completo sobre o assunto a ser tratado, será necessário realizar um estudo dos conceitos gerais na matéria que emanam de convenções internacionais e legislações nacionais; também tendo que analisar os regulamentos existentes em nossa universidade para que possamos trabalhar nos pontos relevantes em sua base.

\section{Palabras clave}

Manual de procedimiento, Universo de obligados, Anticorrupción, Ética Pública.

\section{Keywords}

Procedural Manual, Obligatory universo, Anti-corruption, Public Ethics.

\section{Palavras chave}

Manual de Procedimentos, Universo de Devedores, Anticorrupção, Ética Pública. 


\section{Caracterización del ámbito de gestión y justificación del tema o problema}

Tomaremos dentro un abanico de convenciones, leyes y resoluciones que nos sitúan en un área determinada, pero, indudablemente, es dable reflexionar si nuestra universidad dentro de su autonomía consagrada en el Art. 75 inc. 19 de la Constitucional Nacional, no debe comenzar a ponderar en su estrategia el aumento de los estándares de transparencia y lucha sobre la corrupción.

Así que comenzando desde la cúspide piramidal y la forma de gobierno que ha sido receptada por nuestra Constitución Nacional, el Artículo $1^{\circ}$ establece:

"La Nación Argentina adopta para su gobierno la forma representativa republicana federal",

En su Artículo 36, in fine, correspondiente al capítulo segundo, sección de nuevos derechos y garantías establece que "...Atentará asimismo contra el sistema democrático quien incurriere en grave delito doloso contra el Estado que conlleve enriquecimiento, quedando inhabilitado por el tiempo que las leyes determinen para ocupar cargos o empleos públicos...

El Congreso sancionará una ley sobre ética pública para el ejercicio de la función"1.

El Estado Nacional aprobó mediante ley 26.097, sancionada el 10 de mayo de 2006 y promulgada el 6 de junio del mismo año, la "Convención de las Naciones Unidas contra la Corrupción", y por ley 24.759, sancionada el 4 de diciembre de 1996 y promulgada el 13 de enero de 1997, la "Convención Interamericana contra la Corrupción" entendiendo que ambos instrumentos internacionales fomentan, a través de la transparencia, la lucha contra la corrupción, en pos de garantizar la estabilidad y seguridad de las sociedades.

\footnotetext{
${ }^{1}$ http://servicios.infoleg.gob.ar/infolegInternet/anexos/0-4999/804/norma.htm
} 
Y siendo que la última convención mencionada manifiesta en su preámbulo que “... la corrupción socava la legitimidad de las Instituciones Públicas, atenta contra la sociedad, el orden moral y la justicia, así como el desarrollo integral de los pueblos" y que "la lucha contra la corrupción fortalece las instituciones democráticas, y evita vicios graves en la gestión pública"2.

Que la Convención Interamericana sostiene una "profunda preocupación por los problemas y amenazas que plantea la corrupción para la estabilidad de las instituciones", y convencidos de que el tema requiere un enfoque amplio y multidisciplinario para prevenir y combatir la corrupción.

Es que el Estado Nacional, por ley 27.275, crea la "Ley Nacional de acceso a la información pública", y la misma constituye uno de los requisitos fundamentales de transparencia y configura un elemento de legitimación para el poder administrador estrechamente vinculado con el principio republicano de gobierno.

Así mismo el estado también reglamentó la ley 25.188 sobre "Ética en el ejercicio de la Función Pública", disponiendo el Ministerio de Justicia y Derechos Humanos de la Nación a través de su Resolución 1000/2000 en su artículo $3^{\circ}$ :

"REGISTRO DE FUNCIONARIOS OBLIGADOS. CUMPLIMIENTO. Las áreas de personal, administración o recursos humanos de las jurisdicciones y organismos son responsables de llevar un registro de los funcionarios obligados a la presentación de la Declaración Jurada Patrimonial Integral, en el cual deben dejar constancia del cumplimiento de dicha obligación." 3

\footnotetext{
${ }^{2}$ http://servicios.infoleg.gob.ar/infoleglnternet/anexos/40000-44999/41466/norma.htm 3 http://servicios.infoleg.gob.ar/infoleglnternet/anexos/60000-64999/64623/texact.htm
} 
Desde la perspectiva del mandato de excelencia en sus procederes y los altos estándares delineados por la Universidad, surge la necesidad de ajustar lineamientos respecto esta materia, debido a que en el documento institucional Pensar la Universidad ${ }^{4}$ y en su consecuente Plan Estratégico de Gestión 2018$2022^{5}$, nuestra casa de altos estudios toma de manera amplia la idea de transparencia de sus acciones y de las funciones llevadas adelante por sus agentes.

Así lo demarca el inicio de la estrategia sobre Administración y Gestión: "Objetivo General Administrar y gestionar en forma transparente, eficiente y eficaz, asegurando la distribución equitativa y socialmente responsable de los recursos económicos, de personal, edilicios y logísticos para cumplir con los principios fundamentales de la enseñanza inclusiva, la investigación comprometida y la extensión solidaria" 6 .

Hay que considerar que si bien tanto en Pensar la Universidad como en el Plan Estratégico de Gestión 2018-2022, se menciona la importancia de la transparencia se hace de manera genérica, y que frente a esto la Universidad presenta un déficit estructural y organizacional para establecer una política concreta de transparencia, y anticorrupción.

Lo mencionado hasta aquí, denota la importancia de brindar a la comunidad universitaria la capacidad de distinguir y juzgar los actos de gobierno y las políticas públicas llevadas adelante, ello plasmado a través de un sistema de transparencia que contenga el acceso a la información pública y la lucha contra la corrupción, llevando a consolidar la credibilidad democrática en los actos de

\footnotetext{
${ }^{4}$ http://sedici.unlp.edu.ar/handle/10915/68411

5 https://unlp.edu.ar/plan estrategico

https://unlp.edu.ar/frontend/media/82/11282/7b80557dfc820b35429a3705aac0181e.pd f
} 
gobierno, cuanto mayor transparencia y mayor publicidad de las acciones se establezca, menor será la discrecionalidad, así como menor será la posibilidad de corrupción dentro de la administración.

Que aún no exista un proyecto dentro de la Universidad que englobe la transparencia, la lucha contra la corrupción e incompatibilidad en los cargos públicos, implica una obstaculización para el interés de toda la comunidad universitaria, al cual le debemos la mayor transparencia en nuestras acciones. Es por ello que, como objetivos generales, planteamos la posibilidad de que exista dentro del ámbito universitario un área de contralor y dedicado pura y exclusivamente a estos puntos que hacen a una de las ramas de la transparencia.

Proponemos que se realice por medio de la organización de un área en donde se regule la presentación de las declaraciones juradas a todos los agentes que ejercen el rol de funcionario público, contribuyendo a prevenir el desvío de fondos públicos, el enriquecimiento ilícito (Art. 268 del Código Penal) y el abuso de poder.

\section{Objetivo General}

Proponer una estructuración institucional que comprenda los procesos y herramientas que aseguren la transparencia y la articulación de acciones preventivas de la corrupción o de incompatibilidades en el desarrollo del desempeño de cada miembro de la UNLP.

\section{Objetivos específicos}

- Analizar los actuales procedimientos y herramientas que tengan efecto sobre las temáticas de transparencia y anticorrupción. 
- Procesar críticamente los mecanismos de publicidad y contralor específicos.

- Concentrar y adecuar los instrumentos normativos (ordenanza 293, resolución 849/18, ordenanza 164, y demás resoluciones anexas) en un ordenamiento solidario con la transparencia como núcleo central.

- Producir los lineamientos que conformen un manual de procedimientos que disponga la aplicación de manera uniforme en todas las áreas, dependencias y Unidades Académicas.

- Elaborar estrategias de capacitación específicas, a todos los agentes universitarios a los que de manera transversal le sea aplicable.

\section{Proyecto de intervención}

Como primera medida abordaremos el estudio de la ética en el ejercicio de la función pública, sobre ello tomaremos algunas definiciones y concepto de ética, de lo que se comprende como funcionario público y del buen ejercicio de dicha función en los ámbitos laborales.

Ello así, debido a que para caracterizar el trabajo primero debemos comprender que todo lo referido a lo que se tratará aquí tiene exclusiva aplicación al ámbito de gestión y a quienes detentan el poder de gestionar en nombre de la universidad, ellos son en consecuencia los funcionarios de la misma.

Tomaremos el concepto de corrupción, la lucha contra ello y los órganos de contralor que han intentado entrometerse en la vida universitaria creando una amenaza latente al sentido de autonomía universitaria, realizando a nivel 
nacional un plan denominado "Plan Nacional Anticorrupción 2019-2023"7 que creemos resulta inaplicable a nuestro sistema, pero que ante la negativa y para darle un fundamento debemos reforzar en el interior de nuestra universidad dichos institutos.

Nuestra constitución nacional en su art. 75, inc. 19 establece "que garanticen los principios de gratuidad y equidad de la educación pública estatal y la autonomía y autarquía de las universidades nacionales". La autonomía universitaria es un concepto un tanto complejo de describir, sin embargo referiremos a este como una idea de libertad, de autodeterminación (Rafael I Clark, 2017). Asimismo, al decir de Doberti, la autonomía otorga cierta discrecionalidad a partir de las libertades específicas como la elección de autoridades o la sanción de normas internas (Doberti Juan Ignacio, 2018).

El eje central de esta propuesta de intervención, es llegar a la posibilidad de unificar criterios de trabajo desde la Presidencia de la Universidad, como órgano central de los procedimientos administrativos, hacia todas las Facultades y Dependencias. El objetivo final es no contar con procedimientos desorganizados en cada unidad sino enfatizarse en la creación de un manual que les otorgue a los actores mencionados las pautas procedimentales a seguir.

La creación de un área de anticorrupción y en consecuencia el manual de procedimiento para el área, no solo versa sobre la base de pensar un procedimiento administrativo especial; sino también, de pensar desde el inicio en la creación de un área específica en el tema, dotando a la misma de recursos humanos y materiales.

\footnotetext{
${ }^{7}$ https://www.argentina.gob.ar/anticorrupcion/plan-nacional
} 
Sobre esta base, consideramos esencial contar con personal capacitado en el tema que esté en constante contacto con las Unidades académicas y por sobre todo con los agentes externos que ejercitan un rol principal en esta materia, el cual es hacer de fiscalizador final y aplicador de las normas a nivel nacional y universitario.

En la creación de esta nueva área, dentro de la órbita del organigrama de nuestra Universidad Nacional de La Plata deberá contar con un encargado, con el título suficiente para poder resolver cuestiones legales y técnicas cotidianas y excepcionales de la materia.

El personal deberá entender de manera fluida las necesidades de los funcionarios, la adecuación de su situación de revista, la que luego se verá plasmada por medio de la Declaración Jurada Patrimonial integral, y/o cualquier consulta técnica respecto de cómo encuadrar su situación patrimonial en la misma. De igual forma deberá estar capacitado para el manejo de información sensible y privada del agente y/o funcionario a quien asesore.

\section{Observación en el ámbito Universitario}

La Universidad Nacional de La Plata ha afrontado con total seriedad, exigencia y con la altura que la caracteriza, esta obligación derivada del régimen federal de ética pública integrada dentro del Ministerio de Justicia y Derechos Humanos, la confección del correspondiente listado de los sujetos obligados a la presentación de las Declaraciones Juradas Patrimoniales Integrales.

Los primeros registros completos con los que se cuentan al día de la fecha datan del año 2004, en donde se demuestra un cumplimiento por parte de la Universidad de funcionarios que acceden hasta el cargo de Prosecretarios de Universidad, Secretarios en Facultad y su correspondiente similitud en 
dependencias, esta selección es de tal manera hasta el año 2015, cuando la universidad comienza a entender que es prerrogativa de la misma la delimitación de los sujetos susceptibles a realizar la entrega de la declaración jurada.

Por aquellos tiempos por dictamen de la Dirección General de Asesoría Letrada de nuestra Casa se dispuso ajustar la nómina de los sujetos obligados a realizar la presentación, al texto estricto de la ley; quedando de tal forma el Presidente, los Vicepresidentes, Secretarios de Universidad y Decanos. Por lo que la Universidad reduce la cantidad de agentes sujetos a la presentación de la declaración, llevando el universo a un número final de 78 sujetos obligados. Posterior a dicha decisión comienza una etapa de intercambio de opiniones entre nuestra institución con la Oficina Anticorrupción, quien no consideraba argumento suficiente lo plasmado en el dictamen, obteniendo finalmente en el año 2018 el entendimiento por parte del ente, en sentido de que es prerrogativa de esta Universidad en base a la autonomía universitaria, delimitar quiénes son los sujetos que consideran obligados a presentar la Declaración Jurada.

Los requerimientos de una gestión a nivel nacional que de manera recurrente y constante intenta entrometerse en el mundo universitario, llevó a la necesidad de reafirmar una vez más, los altos estándares con los que trabaja día a día nuestra Universidad Nacional, creando por tal razón una Resolución que amplía el universo de sujetos obligados a la presentación de la Declaración Jurada Patrimonial Integral, que hasta diciembre del año 2017 contaba con tan solo 78 sujetos obligados en donde se encontraban, el presidente de la universidad, el vicepresidente académico, el vicepresidente institucional, los secretarios de universidad, los decanos y vicedecanos y los asimilables directores de colegio; se transforman ahora en 1200 sujetos, ampliando los 
cargos hasta directores ejecutivos de toda la universidad, todo el personal que ostente la máxima categoría del tramo superior del escalafón nodocente, todo el personal que se encuadre en el tramo superior del escalafón nodocente que realice sus actividades dependiendo del área económica y financiera o de la Secretaría de Planeamiento, Obras y Servicios, o de la Secretaría de Administración y Finanzas, directores de institutos, Directorio de la Dirección de Servicio Sociales, comisiones intervinientes en la adjudicación de permisos, concesiones, obras o servicios, Directores de Unidades de Investigación, el Auditor Interno y todo el personal que se desempeñe bajo su dependencia y se encuadre en el tramo superior del escalafón nodocente (Resolución que se adjunta como material anexo).

Sin embargo, este amplio universo recientemente creado, ha generado lagunas administrativas respecto del procedimiento. Esto debido a que, de acuerdo a la ley nacional 25.188 sobre Ética en el ejercicio de la función pública y lucha contra la corrupción, serán las áreas de personal o administrativas las que dispongan o lleven a cabo de manera anual la recepción, control y fiscalización de las Declaraciones Juradas, situación que venía sucediendo de manera considerablemente aceptable, hasta tanto se produjo la ampliación, lo que indefectiblemente llevó a la necesidad de que cada unidad académica y dependencia propusiera su propio universo de obligados, remitiendo la información al área central, que para ese entonces se encontraba aún en la Dirección General de Personal, en tanto luego, y debido a la masiva cantidad de consultas legales y técnicas, el área pasa a establecerse físicamente en la Prosecretaría Legal y Técnica, decisión que concuerda con los fines establecidos en el objeto de la declaración, esto es ya que resulta indispensable contar con un abogado o cuerpo de abogados que entiendan del 
tema. Pase a que también se subsume la Dirección General de Personal, que quedará dentro del organigrama dependiente de la Prosecretaría, por lo cual el área pasó a depender directamente de la Prosecretaría y tener allí mismo su espacio físico, junto al área de transparencia.

El trabajo en resumidas cuentas, luego del diagnóstico realizado precedentemente y haber estudiado el objeto, intenta planificar (Mathus Carlos, 1987) un área que en el día de hoy efectivamente se encuentra en funcionamiento, pero que, luego de las reformas mencionadas ha crecido exponencialmente y que hoy en día, ha se muestra insuficiente para atender adecuadamente la creciente demanda con los altos estándares de eficiencia que la UNLP fija para sus procedimientos en cuanto a espacio físico y a personal. Por lo que este trabajo es de aplicación junto con la creación de un área y de aplicación de un manual de procedimientos que sea utilizado tanto por el área creada como por todos los representantes de dependencias y unidades académicas.

Tal como se mencionó precedentemente, el área aún no oficializada como tal ha tenido un crecimiento excesivo en el último periodo, debiendo no solo dar respuestas técnicas correspondiente al área de anticorrupción, sino también en la evacuación de dudas y consultas respecto diferentes cuestiones abarcativas de varias áreas de la universidad.

Mencionaremos a continuación su justificativo:

$\checkmark$ En primer término, fundamentalmente, las facultades y dependencias están en constante consulta con el área central, ya sea por situaciones individuales o por la totalidad del universo: por lo que, si nosotros con un simple número de DNI obtenemos toda la información procesada del área de anticorrupción 
desde el año 2004 hasta la fecha, podremos en tan solo instantes evacuar las dudas que se consideren necesarias.

Como segundo punto porque un agente (siendo que la mayoría de ellos son cargos de gestión) puede haberse dado de alta o baja en varias oportunidades, y acceder a todas sus declaraciones para poder encuadrar su situación resulta casi imposible siendo que no contamos con ellas a nuestro alcance.

$\checkmark$ Por último, dentro del área, tarea que aún no ha sido explicada, expedimos certificados de realización de declaraciones juradas, con el número de transacción que le corresponde a la última presentación realizada. Esta situación, ocurrida en varias oportunidades sucede por demanda de la Administración Federal de Ingresos Públicos, que al cruce de datos solicita a los agentes la aclaración o reenvío de Declaración Jurada.

Resulta menester aclarar, que las Declaraciones Juradas de los años ya transcurridos, no están al alcance de nuestras manos, sino que se encuentran en un archivo en el edifico anexo a la presidencia, por lo que la búsqueda de alguna declaración anterior al año en curso resulta sumamente engorrosa.

Ello debido a que el área, el cual hoy en día cuenta con una única persona que atiende la materia, atiende una inmensa cantidad de consultas de carácter jurídico, por lo que el asesoramiento que se brinda es constante.

\section{Desarrollo del proyecto de intervención}

Se propone como recursos necesarios crear un área específica establecida dentro del organigrama de nuestra universidad, para generar de tal manera la posibilidad de reorganización, este área debería ubicarse dentro de la Secretaría de Asuntos Jurídico Legales, por la temática requerida, entendiendo que la Oficina Anticorrupción como órgano de control se encuentra actualmente 
dirigida por un abogado inmerso dentro del ámbito jurídico de la Nación, puede estar bajo la dependencia de la Prosecretaría Legal y Técnica. Sin embargo, es indispensable que se cree un área con un responsable determinado que podría tener carácter de Director y personal afectado a la misma.

Como objeto principal se acerca la propuesta de crear un área específica en el tema, en donde se establezca comunicación permanente con el personal afectado en cada unidad y/o dependencia al tratamiento de la materia. Dentro de la creación del área las tareas fundamentales a realizar serían la recepción de las Declaraciones Juradas Patrimoniales Integrales, la fiscalización y contralor del trabajo realizado en las facultades y dependencias, el contacto directo con la Oficina Anticorrupción como órgano central y las intimaciones correspondientes a los sujetos que no han dado cumplimiento a la obligación en los plazos fijados por ley.

\section{Elaboración de un manual de procedimiento}

El Manual de Procedimiento corresponde a un proyecto que emana de la necesidad de encontrar una organización y un procedimiento común frente a la conformación del llamado universo de obligados, y a los cambios que dentro del mismo se producen, estos pueden ser altas o bajas y promociones para el caso de los trabajadores nodocentes.

\section{Reflexiones finales}

Hemos visto en el transcurso del presente trabajo de investigación final diferentes conceptos y organismos que dan a la transparencia y al acceso a la información como también a la lucha contra la corrupción una importancia trascendental, hemos señalado organismos internacionales como la OEA, las 
diferentes convenciones interamericanas, que de manera constante plantean en sus propósitos la necesidad de erradicar la corrupción de los estados, como también pregonan la obligación de cumplimiento de los derechos a la libertad de expresión y por tal al derecho a la información, puntos que están de una manera u otra conectados, ya que en todas las normativas que hemos nombrado precedentemente y serán anexadas al trabajo nos indica que un estado que publica sus actos, un estado que cumplimenta con el derecho a la información, que tiene transparencia, se entendería como un estado que tenga menor corrupción.

No existe a nivel nacional, muchas universidades que traten los temas de anticorrupción. Es de observar que nuestra casa de altos estudios siempre ha sido pionera en interminable cantidad de cuestiones, y puntualmente el acceso a la información pública y la lucha contra la corrupción debe ser otro punto en cuestión.

Tomar medidas relativas a la lucha contra la corrupción, y llevar a nuestra institución a los máximos estándares de transparencia es un punto notable a trabajar.

He intentado en el presente trabajo, plasmar algunos puntos de partida posibles a adaptar a la vida universitaria, para generar unificadamente en todos los ámbitos de la universidad políticas y procedimientos que nos lleven a dar un paso más en la lucha contra la corrupción.

\section{Bibliografía}

- Balbín, Carlos: Manual de Derecho Administrativo, 3er. Edición, Thomson Reuters - La Ley, Buenos Aires 2015. 
- Bobbio Norberto. El futuro de la democracia, Fondo de Cultura Económica, México 1991.

- Clark Rafael I.: Autonomía Universitaria y Control Judicial.: Ad-Hoc SRL., Buenos Aires, 2017.

- Comisión Interamericana de Derechos Humanos: El derecho de acceso a la información pública en el marco jurídico Interamericano. Comisión Interamericana de Derecho Humanos - OEA, (2010).

- Doberti Juan Ignacio: La organización Académica en la Universidad, capítulo 2, EUDEBA, Buenos Aires, 2018.

- Fayt, Carlos S.: Derecho Político, Tomo I 9na edición, Ediciones Depalma, Buenos Aires, 1995.

- Fayt, Carlos S.: Derecho Político, Tomo II 9na edición, Ediciones Depalma, Buenos Aires, 1995.

- Gordillo, Agustín: Teoría General, Tomo I, Capitulo XVI. 10ª ed., Buenos Aires, Fundación de Derecho Administrativo., 2009

- Ivanega, M. M: La responsabilidad de los funcionarios públicos. Revista Virtual UNAM, 158.

- Marcela Basterra, Acceso a la Información Pública, 1er edición, Editorial Astrea, Ciudad autónoma de buenos aires, 2017.

- Marienhoff, Miguel. S.: Tratado de derecho administrativo. Tomo I, 5 Ed, LexisNexis Albeledo Perrot, Buenos Aires, 2003.

- Marienhoff, Miguel. S.: Tratado de derecho administrativo - T III B. $4^{\circ} \mathrm{Ed}$, LexisNexis Albeledo Perrot, Buenos Aires. 1998

- Mathus Carlos: Política, planificación y gobierno. Caracas: FUNDACION ALTADIR.1987

- Montesquieu: El espíritu de las leyes. Tomo I, libro II y III, Madrid, 1821. 
- Pérez Lindo Augusto, La Educación Superior Argentina (1983-2005) diagnóstico y perspectiva, Eudeba, Buenos Aires, 2017.

- Perrino, Pablo E., La Responsabilidad del estado y los funcionarios Públicos, Thomson Reuters - La Ley, Ciudad Autónoma de Buenos Aires, 2015.

- Tauber, Fernando, Pensar la Universidad.

- Real Academia Española. (2019).

- Ziulu, Adolfo. G: Derecho Constitucional, Principios y Derechos Constitucionales, Tomo I, Buenos Aires, Ediciones Depalma, 1996. 